\title{
Development and study of a microwave reflex-radar level gauge of the nuclear reactor coolant ${ }^{*}$
}

\author{
Vladimir I. Melnikov ${ }^{1}$, Vadim V. Ivanov ${ }^{1}$, Ivan A. Teplyashin ${ }^{1}$, Mikhail A. Timonin ${ }^{1}$ \\ 1 Nizhny Novgorod State Technical University n.a. R.E. Alekseev, 24 Minin str., Nizhny Novgorod, 603600 Russia \\ Corresponding author: Vladimir I. Melnikov (melnikov@nntu.ru)
}

Academic editor: Boris Balakin • Received 11 August 2018 Accepted 13 November 2018 Published 7 December 2018

Citation: Melnikov VI, Ivanov VV, Teplyashin IA, Timonin MA (2018) Development and study of a microwave reflex-radar level gauge of the nuclear reactor coolant. Nuclear Energy and Technology 4(3): 185-190. https://doi.org/10.3897/nucet.4.31857

\begin{abstract}
The article considers the design of a microwave reflex-radar level gauge of the nuclear reactor coolant. The main advantage of the reflex-radar measurement principle is that it does not affect the accuracy of measuring the level of bubbles present, coolant condensation and boiling, changes in its pressure as well as temperature and density. In addition, the measuring transmitter design is quite simple.

In this level gauge, a microwave waveguide made as a coaxial line is used as a transducer (measuring probe). The probe consists of a steel pipe with an external diameter of $20 \mathrm{~mm}$ and a central electrode: it is located vertically and immersed in a controlled coolant. The probe wave resistance is $50 \mathrm{ohms}$. The device electrical diagram is presented. The oscillograms of the received signals and the basic relationships explaining the level gauge operation are given. The signals of the coaxial measuring probe are studied in a fluid with a variable dielectric constant. The results of an experimental study of the level gauge operation in a water coolant at high parameters are given: at pressures up to $10 \mathrm{MPa}$ and temperatures up to $310^{\circ} \mathrm{C}$. It is shown that the device maintains its functional stability under these conditions. The level gauge's readings practically need not be corrected when the coolant's thermophysical properties change. The device is intended for use in the control and management systems of nuclear power plants as well as in fuel reprocessing plants.
\end{abstract}

\section{Keywords}

Microwave reflex-radar level gauge, high pressure water coolant, measuring probe, nuclear reactor, power-generating equipment

\section{Introduction}

Measuring the coolant level in a nuclear power plant (NPP) is a complex technical task. The level gauge should work reliably for a long time in extreme operational conditions, i.e., at high pressure, temperature and radiation levels typical for nuclear power equipment.

Despite the complexity of the task, a very limited number of instruments are used in the nuclear power industry, in particular, reactor level control devices based on the hydrosta- tic principle. It seems that ultrasonic level gauges, in which steel waveguides are used to conduct acoustic signals into internal circuit volumes, have good prospects. In addition, thermoelectric and conductometric level gauges are being developed. It should be noted that the problem of measuring the level of process media is also relevant in other branches of the nuclear industry, as evidenced by the significant variety of created level measuring methods and technical tools.

The most important tasks to be solved in the development of gauges include instrument reliability, reduction

${ }^{*}$ Russian text published: Izvestiya vuzov. Yadernaya Energetika (ISSN 0204-3327), 2018, n. 2, pp. 15-24. 
(exclusion) of the influence of various interfering factors on measurement results, longevity and durability in media with variable and extreme physical parameters.

In recent years, level gauges for detecting controlled media by microwave pulses have significantly evolved. Their appearance is associated primarily with the development of microwave technology and the advent of relatively cheap high-speed microelectronics, which allowed miniaturization of information processing systems, tuning and diagnostics of level gauges. These most advanced and sophisticated level control devices became widespread only at the beginning of the new millennium.

Reflex-radar level gauges are based on the time domain reflectometry (TDR) method used for damage testing in communication lines (Glebovich et al. 1984, Vorontsov and Frolov 1985, Tarasov 2018, Jones et al. 2002).

Their main advantages are the simplicity of the technical implementation of the primary sensor, ensuring its high long-term stability when exposed to an environment with high temperature and pressure, as well as the fact that the measured value is the time interval, i.e., the electrical pulse travel time to the gas-liquid interface and back that can be measured with high accuracy and hardly at all depends on various technological parameters of the coolant (temperature, pressure, vapor concentration, etc.).

The main component of a reflex-radar gauge is a measuring probe, which is a microwave waveguide located vertically and partially immersed in a controlled medium. Waveguides in the form of a coaxial or two-wire line are used (Weiss and Knochel 1997, Cataldo et al. 2007).

At the top of the probe, the electronic circuit generates short electromagnetic pulses that propagate down through the waveguide at a speed close to the speed of light. When a pulse reaches the surface of a liquid having a higher dielectric constant than that of the air in which it has propagated before this, the pulse is reflected. The high-speed circuit determines the pulse propagation time from the moment of its sending to receiving and calculates the fluid level. The greater the dielectric constant of a fluid, the stronger this reflection will be. It is important that the propagation of an electromagnetic pulse is not significantly affected by changes in pressure and temperature, the presence of foam, steam, and other environmental factors (Trenkal and Loschilov 2016, Jun et al. 2013, Xiaowen et al. 2013).

Note that all designs of reflex-radar level gauges presented in technical and patent literature and advertising sources are products of foreign companies (Dozer 1968, Hook 1998, Reimelt and Schroth 2002, Gravel and Fandrey 2005, Instructions 2018). Domestic devices are absent, so the cost of such devices on the Russian market is significant and reaches several thousand dollars.

The paper presents the results of research aimed at creating and testing domestic microwave reflex-radar level gauges of the water coolant for use in nuclear power engineering.

The main problems in developing these devices are (1) selecting a method for accurate measurements of very short time intervals (within nanoseconds) and (2) creating a heat-resistant, high-pressure sealing assembly for conducting electromagnetic microwave signals inside the equipment of the primary and secondary circuits.

\section{Level gauge structural design}

The level gauge consists of a measuring probe, made as a coaxial line, a delay line and a secondary electronic unit. We have developed a device design with a coaxial probe $0.7 \mathrm{~m}$ long and wave resistance $50 \mathrm{ohms}$. A $1.5 \mathrm{~m}$ long coaxial cable was used as a delay line (Fig. 1).

The probe is made of a pipe with a diameter of $20^{\prime} 2$ $\mathrm{mm}$. A rod with a diameter of $7 \mathrm{~mm}$ was used as the central electrode. Compaction and electrical insulation of the central electrode in the upper part of the probes are made using a node based on heat-resistant ceramics. A fitting with a $3 / 4$ " thread connection is used to fasten and seal the probe in the vessel. The probe body at the bottom, at the free end, is electrically connected to the central electrode, forming a short-circuited line. The central electrode is spaced by suspension elements made of pot stone bushings.

The structural chart of the device and the signals at the entrance to the comparator at different water levels are shown in Fig. 2.

The electronic unit includes a square-wave pulse generator connected through a $50 \mathrm{ohm}$ matching resistor and a delay line to the probe; a gated comparator, the second input of which is supplied with voltage from the level adjus-

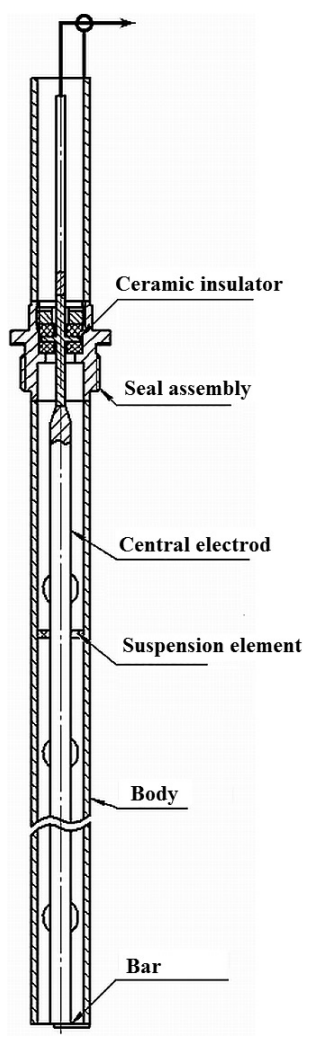

Figure 1. Measuring probe. 


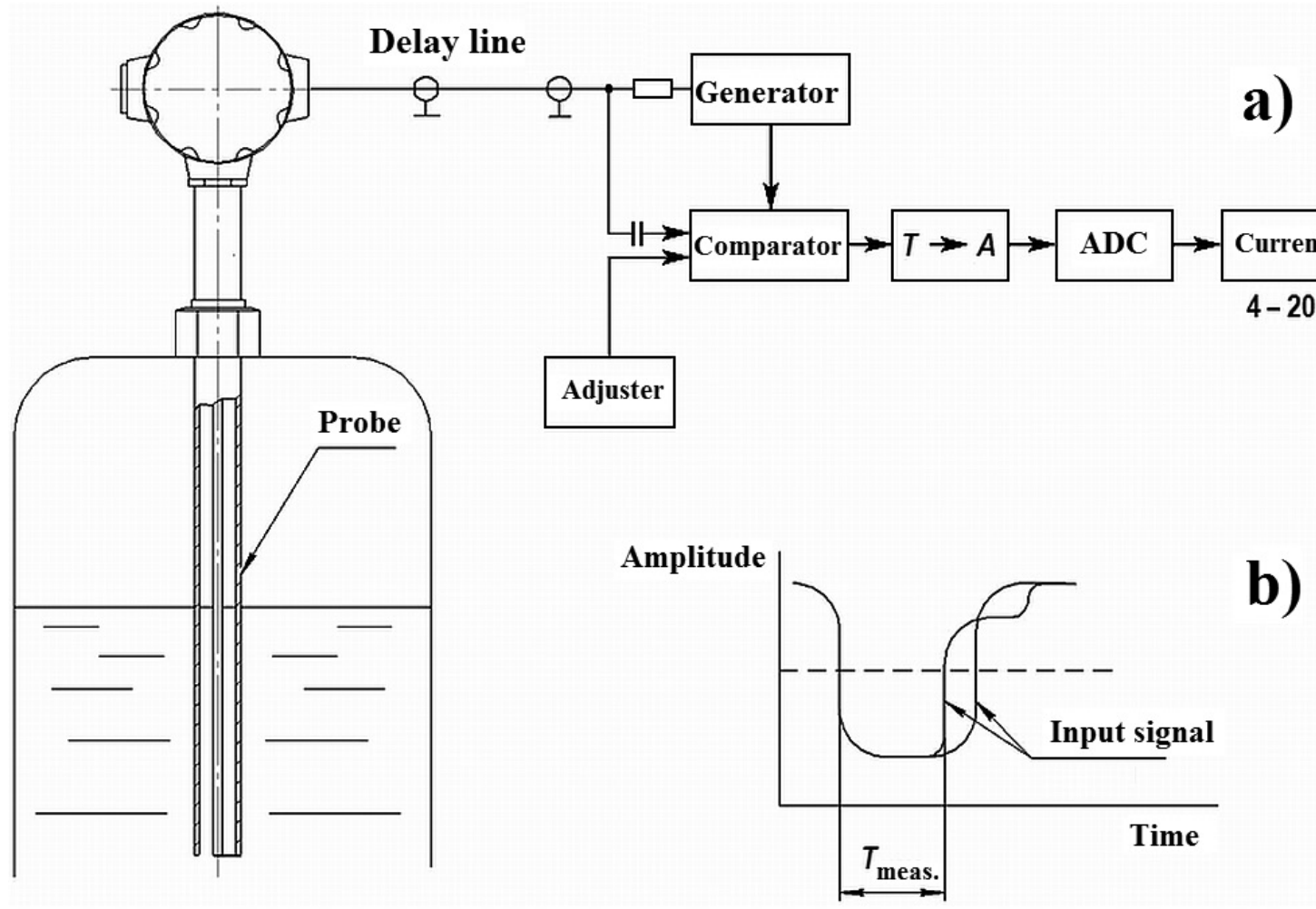

Figure 2. Device structural chart (a) and comparator input signals (b).

ter; a pulse length-amplitude converter; an analog-to-digital converter; and an amplitude-current converter.

In the output stage of the generator, a transistor with a boundary frequency of $7 \mathrm{GHz}$ is used, the comparator has a characteristic response time of $2.7 \mathrm{~ns}$, and the PIC series microprocessor performs the functions of the rest of the circuit.

The device works as follows. The negative pulse front with an amplitude of $U_{0}$ passes from the generator output through the resistor and the delay line to the probe, where it generates a microwave electromagnetic wave in the space between the body and the central electrode. This wave propagates down the probe to the point of closure of the central electrode with the body and is reflected back. As a result, a rectangular pulse is formed at the output delay line output, which is a superposition of the sent and received waves. This rectangular pulse shape is due to the fact that the reflected pulse returns in antiphase to the sent one, as a result of which they are mutually compensated with a delay equal to the running time of the signal along the delay line and the probe to the phase line and back. Then the received pulse is fed to the input of the high-speed comparator, to another input of which voltage is supplied from the level adjuster output. The nanosecond pulse from the output of the comparator is converted by means of a "pulse length-amplitude" converter into a voltage level proportional to the input pulse length, which is then converted into a digital code by means of an ADC. Further, the circuit produces an output current signal at a given load, proportional to the received digital code (level).

\section{Probe signals and level gauge testing}

The pulse amplitude at the probe output (at the comparator connection point) is half the amplitude of the pulse generated by the generator. It is determined by the ratio

$$
U=U_{0} Z_{0} /\left(Z_{0}+R_{0}\right),
$$

where $U_{0}$ is the pulse amplitude at the generator output (about $3.6 \mathrm{~V}$ ); $Z_{0}$ is the delay line wave resistance (50 $\mathrm{ohms}) ; R_{0}$ is the matching resistor resistance (50 ohms).

When the probe is immersed in water, part of the electromagnetic wave energy is reflected from the air-fluid interface, another part of the wave energy goes further to the probe shorted end, is also reflected and returns back (Cataldo et al. 2011).

The wave reflected from the air-fluid interface comes ahead (relative to the unfilled probe). The wave reflected from the probe tip, on the contrary, returns with a delay. This delay value results from the wave velocity decline due to the fact that the probe internal volume is filled with a dielectric (controlled fluid).

The amplitude of the wave reflected from the air-fluid interface is determined by the reflection coefficient, which is equal to (Nemarich 2001)

$$
K=(\sqrt{\varepsilon}-1) /(\sqrt{\varepsilon}+1),
$$

where is the fluid relative dielectric constant. 
In the temperature range of $20-350^{\circ} \mathrm{C}$, the water coolant relative dielectric constant varies from about 80 to 15 (Teploenergetika i teplotechnika 1999), while the reflection coefficient varies slightly, from 0.8 to 0.59 .

In the water coolant, most of the electromagnetic wave energy is reflected from the air-water interface, not reaching the probe tip, which is clearly seen on the oscillogram (Fig. 3). At the same time, the delay of the signal reflected from the probe tip $\left(\mathrm{T}_{\mathrm{p}}\right)$ is quite significant due to the high value of the water dielectric constant.

The fluid level is determined from the ratio

$$
H=C\left(T_{1}-T_{2}\right) / 2,
$$

where $C$ is the propagation velocity of an electromagnetic wave in the upper, air-filled part of the probe.

The wave velocity in the probe depends on the dielectric constant of the fluid filling the interelectrode space:

$$
C_{\mathrm{Fl}}=C \varepsilon^{1 / 2} \text {. }
$$

It follows that there is a second variant of measuring the fluid level by the delay value of the pulse reflected from the probe tip $T_{\mathrm{P}}$ :

$$
H=C_{\mathrm{Fl}}\left(T_{\mathrm{P}}-T_{1}\right) / 2 .
$$

But it should be borne in mind that the $T_{\mathrm{P}}$ value is significantly influenced by the dielectric constant of the controlled medium.

The oscillogram (Fig. 4) shows the signals of the probe in air and immersed in different fluids by about half its length (fluids: water under normal conditions $=80$ and acetone $=21$ imitating water on the saturation line at a temperature of approximately $310^{\circ} \mathrm{C}$ ).

\section{Experimental tests}

Initially, the sample level gauge was tested in a bench-testing unit under normal conditions by immersing the sensitive element of the sensor (probe) in water. The output current varied in proportion to the fluid level (Fig. 5). The average squared deviation of the measured level from the actual one did not exceed $0.25 \%$.

Experiments at high parameters were carried out in the coolant on the saturation line with variations in pressure from 0.1 to $11 \mathrm{MPa}$. The experimental unit (Melnikov et al. 2015) was a robust hermetically sealed and electrically heated pipe, in which the gauge was placed. The heater power was regulated by a laboratory autotransformer. The maximum coolant temperature reached $310^{\circ} \mathrm{C}$.

Before the experiment, the level gauge was installed in the pipe, the system was filled with water to the $460 \mathrm{~mm}$ mark from the lower end of the pipe, then the installation was sealed. After the measuring circuit was prepared, a voltage was supplied to the electric heater. The temperature in the system gradually increased at a rate of about

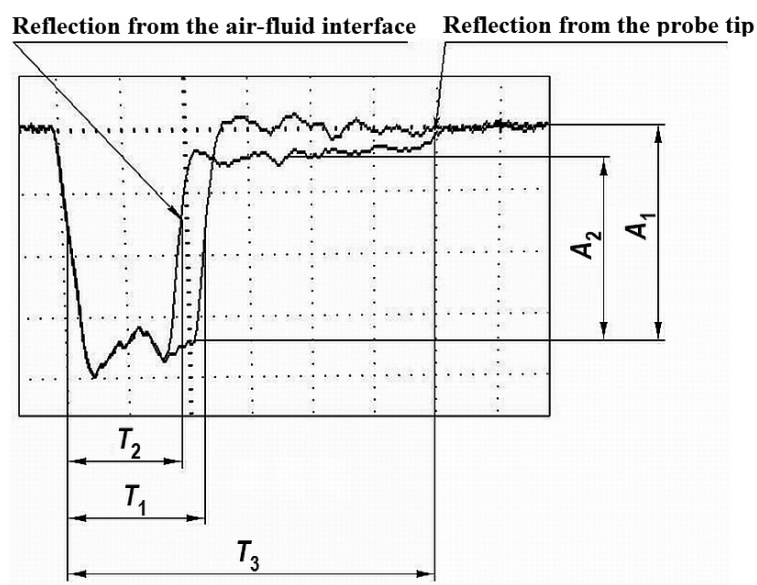

Figure 3. Combined oscillograms of signals at the probe output in air and completely immersed in water under normal conditions (vertical division value: $0.5 \mathrm{~V} /$ cell., horizontal division value: $10 \mathrm{~ns} /$ cell.): $T_{1}$ is the pulse duration in air; $T_{2}$ is the duration of the pulse reflected from the air-water interface; $T_{3}$ is the duration of the pulse reflected from the probe tip in water; $A_{1}$ is the sent pulse amplitude; $A_{2}$ is the amplitude of the pulse reflected from the air-water interface.

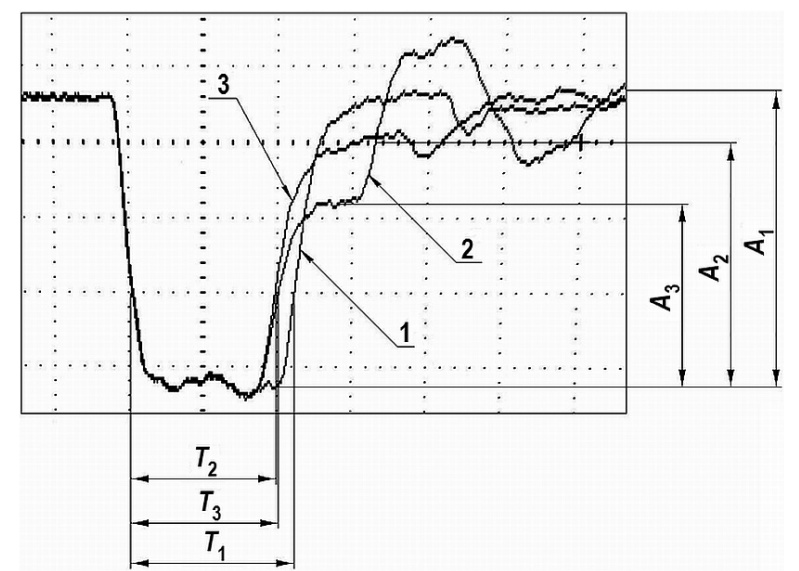

Figure 4. Combined oscillograms of signals at the probe output under normal conditions in air (1), in acetone (2) and water (3), the probe immersion depth in a fluid is $390 \mathrm{~mm}$ (vertical division value: $0.5 \mathrm{~V} /$ cell, horizontal division value: $10 \mathrm{~ns} /$ cell). $T_{1}$ is the pulse duration in air; $T_{2}$ is the duration of the pulse reflected from the air-water interface; $T_{\mathrm{P}}$ is the duration of the pulse reflected from the air-acetone interface; $A_{1}$ is the sent pulse amplitude; $A_{2}$ is the amplitude of the pulse reflected from the air-water interface; $A_{3}$ is the amplitude of the pulse reflected from the air-acetone interface.

$7{ }^{\circ} \mathrm{C} / \mathrm{min}$ to about $310^{\circ} \mathrm{C}$. At the same time, the pressure increased to about $11 \mathrm{MPa}$. Measurements were made of the coolant temperature and pressure inside the pipe as well as the gauge output current.

While the pipe was heated inside, equilibrium was established between the coolant liquid and vapor phases on the saturation line due to partial evaporation of water at a constant volume. At the same time, as the temperature rises, the pressure and water level also increase. We de- 


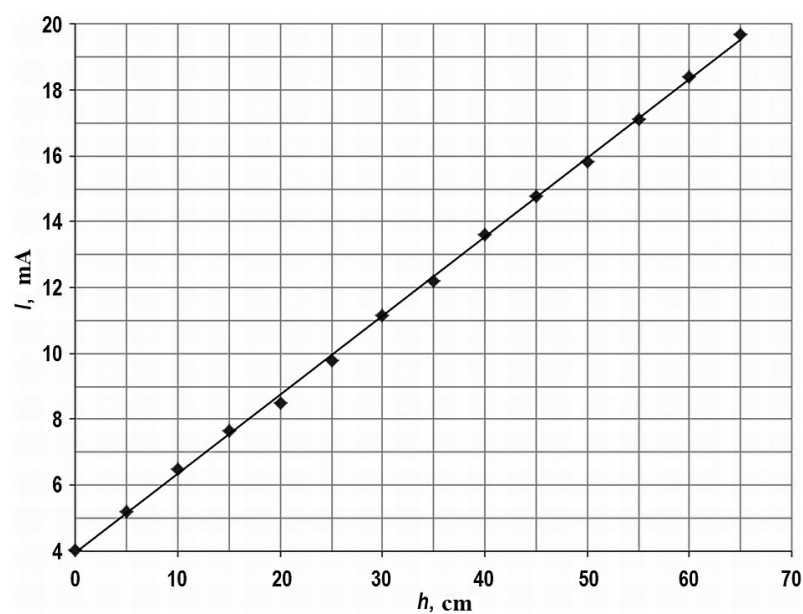

Figure 5. Gauge calibration characteristic in water under normal conditions.

termined the temperature dependences of the water level changes on the saturation line: the calculated and measured using the reflex-radar level gauge (Fig. 6).

At high temperatures, there is a discrepancy between the measured coolant level and the calculated value of about $20 \mathrm{~mm}$ (3\% of the measuring range) downwards. The analysis showed that the reason for this may be a decrease in the coolant dielectric constant as the temperature increases. As $\varepsilon$ decreases, a reflected microwave energy pulse requires a larger fluid layer, which manifests itself as an apparent level decrease.

The level gauge readings can be adjusted by taking into account changes in the coolant dielectric constant. As follows from the expression (2)

$$
\mathrm{e}=[(1+K) /(1-K)]^{2},
$$

where the reflection coefficient $K$ is determined by the shape of the pulse at the probe output.

In particular, for the water coolant at a temperature of $310{ }^{\circ} \mathrm{C}, K=A_{3} / A_{1} \gg 0.64$ (see Fig. 4), whence it follows that $\mathrm{e} » 20$.

\section{References}

- Cataldo A, De Benedetto E, Cannazza G (2011) Broadband Reflectometry for Enhanced Diagnostics and Monitoring Applications. Springer Science \& Business Media, 150 pp. https://doi. org/10.1007/978-3-642-20233-9

- Cataldo A, Tarricone L, Attivissimo F, Trotta A (2007) A TDR Method for Real-Time Monitoring of Liquids. IEEE Transactions on Instrumentation and Measurement 56: 582-595. https://doi. org/10.1109/TIM.2007.903596

- Dozer BE (1968) Selfcalibrating liquid-level measuring device. Patent US, no. 3398578.

- Glebovich GV, Andriyanov AV, Vvedencky YV, Kovalev IP, Krylov VV, Ryabinin YA (1984) Investigation of objects using picosecond pulses. Moscow. Radio i Svyaz Publ., 256 pp. [In Russian]

- Gravel JL, Fandrey MC (2005) Tank seal for guided wave radar level measurement Patent US, no. 7255002.

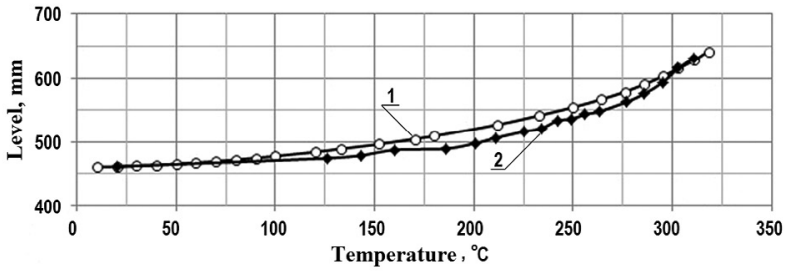

Figure 6. Temperature-dependent coolant level changes on the saturation line: 1) calculated; 2) measured

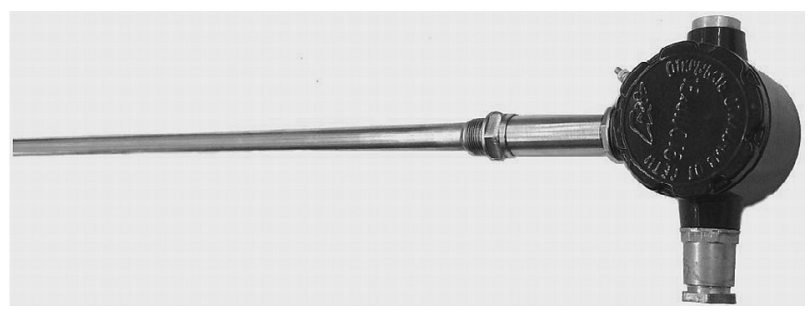

Figure 7. Microwave reflex-radar level gauge.

Figure 7 shows the exterior of a microwave reflex-radar level gauge manufactured by the "Acoustic Measuring Systems-NN" Company.

\section{Conclusion}

The article presents the results of studies of physical processes in the microwave path of a microwave reflex-radar level gauge. The design of the device is considered and its bench tests are carried out in the water coolant medium. It is confirmed that the developed level gauge can be used to control the medium at temperatures up to $310{ }^{\circ} \mathrm{C}$ and pressures up to $11 \mathrm{MPa}$. The device can be used in various areas of industry and national economy, in particular, to control the coolant level in the instrumentation and control systems of nuclear power plants as well as at spent fuel reprocessing plants.

- Hook WR (1998) Apparatus and methods for time domain reflectometry. Patent US, no. 5726578.

- Instructions for installing and operating the BM100 level transmitter company KROHNE (2018). www.ste.ru/krohne/pdf/rus/russ_op_ manualBM100.pdf [accessed Mar 10 2018] [In Russian]

- Jones SB, Wraith JM, Or D (2002) Time domain reflectometry measurement principles and application. Hydrol Process 16: 141-153. https://doi.org/10.1002/hyp.513

- Jun G, Huigin Z, Hongyu Y, Hui Z (2013) Design of guided wave radar level meter based on equivalent time sampling. Proc. of the IEEE Int. Conf. "Communications, Circuit and Systems (ICCCAS)". Chengdu, China, 15-17 Nov. 2: 139-142.

- Melnikov VI, Ivanov VV, Teplyashin IA, Timonin MA (2015) The study of ultrasonic waveguide level gage of nuclear reactor coolant 
on the basic of reflex-radar principle. Izvestia visshikh uchebnikh zavedeniy. Yadernaya energetika, 4: 26-35. [In Russian]

- Nemarich CP (2001) Time domain reflectometry liquid level sensors. IEEE instrumentation \& measurement magazine 4(4): 40-44. https://doi.org/10.1109/5289.975464

- Reimelt R, Schroth H (2002) Device for determining and/or monitoring the fill level of a product in a container. Patent DE, no. 1010945.

- Tarasov NA (2018) Using the method of pulse reflectometry for determination of damage of cable lines. Available at: http://reis.narod. ru/metod.htm [accessed Mar 10 2018]. [In Russian]

- Teploenergetika i teplotechnika (1999) Vol. 1. General questions. Section 7. Physico-chemical properties and technology solutions. Moscow. MEI Publ., 528 pp.
Trenkal EI, Loschilov AG (2016) Measurement of liquid levels by pulse reflectometry (review). Proc. of the Tomsk GUSUR, 19(4): 67-73. [In Russian]

- Vorontsov AS, Frolov PA (1985) Pulse measurement of coaxial communication cables. Moscow. Radio i Svyaz Publ., 96 pp. [In Russian]

- Weiss M, Knochel R (1997) Novel method of measuring impurity levels in liquid tanks. 1997 IEEE MTT-S International Microwave Symposium Digest 3: 1651-1654. https://doi.org/10.1109/ MWSYM.1997.596709

- Xiaowen X, Zonghui L, Junkai G, Hongmin G (2013) The principle and simulation of the TDR test. Proc. of the IEEE Int. Conf. "Microwave Techn. and Comp. Electromagnetics”. Qingdao, China, 25-28 Aug.: 47-51. https://doi.org/10.1109/ICMTCE.2013.6812478 